\title{
Indoor Navigation System based on Passive RFID Transponder with Digital Compass for Visually Impaired People
}

\author{
A. M. Kassim, T. Yasuno and H. Suzuki \\ Graduate School of Tokushima University, \\ 2-1 Minamijosanjima, Tokushima, 770-8506, JAPAN
}

\author{
H. I. Jaafar and M. S. M. Aras \\ Universiti Teknikal Malaysia Melaka \\ Hang Tuah Jaya, Durian Tunggal, 76100 Melaka, MALAYSIA
}

\begin{abstract}
Conventionally, visually impaired people using white cane or guide dog for traveling to desired destination. However, they could not identify their surround easily. Hence, this paper describes the development of navigation system which is applied to guide the visually impaired people at an indoor environment. To provide an efficient and user-friendly navigation tools, a navigation device is developed by using passive radio frequency identification (RFID) transponders which are mounted on the floor such as on tactile paving to build such as RFID networks. The developed navigation system is equipped with a digital compass to facilitate the visually impaired people to walk properly at right direction especially when turning process. The idea of positioning and localization with the digital compass and direction guiding through voice commands is implemented in this system. Some experiments also done which is focused on the calibration of the digital compass and relocates the visually impaired people back to the right route if they are out of the direction. Besides, a comparison between two subjects which are human and a mobile robot is made to check the validity of the developed navigation system. As the result, the traveling speed of human and mobile robot is obtained from the experiment. This project is beneficial to visually impaired people because the navigation device designed with voice commands will help them to have a better experience, safer and comfortable travel.
\end{abstract}

Keywords-navigation system, passive RFID, digital compass, visually impaired people

\section{INTRODUCTION}

World Health Organization (WHO) has released the statistics in 2014 that shows $10 \%$ of the world's population have a disability, with $80 \%$ of them located in developing countries. This global data on visual impairments shows the people with visual impairment globally is around 285 million which 39 million are fully blind and 246 million are having low vision problem[1]. There are great numbers of people worldwide who have encountered vision loss including Malaysia. This total figure does not reflect to the real number of a people with disabilities in this world since the visually impaired people are cannot independently travel by themselves.

Moreover, a disability that may involve additional danger to the individual is blindness. Conventionally, they rely on a white cane or a guide dog to assist them in reaching the desired destination safely. It has been decades since visually impaired people use the white cane as their most common and affordable assistive tool to detect obstacles and path surrounding them. Difficulties still occur when using the white cane for visually impaired people as they are only able to detect path and obstacles from the front by swinging the white cane at the same time trying to feel the tip of the white cane that touches the ground.

However, this approach is useful if the passage to the desired place is already known to them. It also becomes troublesome once the destination is newly constructed and not implemented the universal design, especially on visually impaired people. They do not receive enough information with only the tip of the white cane as feedback. Lack of aid signs built for visually impaired people seems to be one of the difficulties for them. It is hard and nearly impossible to recognize the place by themselves and travel from one to another destination without proper navigation tools. Therefore, the advanced technology developed by researcher benefits the visually impaired people to move independently.

In these two decades, there are quite some equipments, tools or robots which have been developed by the researchers in this world to assist the visually impaired people. The assistive and rehabilitation technologies that have been researched and built are such as GuideCane[2], NavBelt[3], My 2nd Eye[4], SMART EYE [5], [6], and others. Besides that, one of the developed mobile robots for the blind which is designed to help the blind in shopping malls [7]. Without the state of the art of these technologies, the visually impaired people only counts on the conventional white cane to detect surrounding obstacles and sense the road in front of them.

The arrangement of this paper is done as follows where Section 1 presents an introduction on problem and challenge that have been faced by visually impaired people. Section 2 expresses previous studies related to the travel aid for visually impaired people. Besides, section 3 deliberates on the developed navigation system with the proposed control system for navigation purpose. Section 4 discloses on experimental setup involved in this study while Section 5 elaborates the results that obtained through the developed navigation system and the proposed control approach, and lastly the conclusion and future tasks of this study.

\section{RELATED WORKS}

The design challenges for the blind navigation device are real-time guidance, portability, power limitations, appropriate interface, and continuous availability, no dependence on in- 
frastructure, low cost solution and minimal training [8]. There are several designs to help visually impaired people to achieve self-independence traveling at indoor environment. Location technology such as infrared data association (IrDA), RFID, Bluetooth or Wi-Fi has been developed to help them travel during indoors with contextual information or sound navigation [9] .

In addition, the usage of Global Positioning System (GPS) device also can help to guide the visually impaired people in an outdoor environment. Since GPS cannot function properly in indoor space, other researchers present the solution by using IrDA technology which works as detector to guide in indoor environment[10]. The Drishti system is the combination of GPS for outdoor navigation and ultrasonic sensor for indoor navigation[11]. One of the problems about GPS is the error with the measurement taken especially inside the tall buildings [12].

Furthermore, BLI-NAV a blind navigation system designed which consists of GPS receiver and path detector. Both devices used to detect user's location and determine the shortest route to destination. Voice command is given throughout the travel. Path algorithm is used to determine the shortest distance from start point to end point together with path detector. Moreover, user is able to avoid obstacles while traveling [13]. This system gave better results in real time performance and improves the efficiency of blind travel at indoor environment.

On the other hand, Pocket-PC based Electronic Travel Aid (ETA) proposed to help visually impaired people to travel at indoor environment. Pocket-PC will alert the user when near the obstacles through warning audio [14]. An ultrasonic navigation device for visually impaired people is designed. The micro-controller built in the device can guide the user in which route should be taken through speech output. Besides, the device helps to reduce navigation difficulties and an obstacles detection using ultrasounds and vibrators. Ultrasonic range sensor is used to detect surrounding obstacles and electronic compass is used for direction navigation purpose. Stereoscopic sonar system is also used to detect nearest obstacles and it feeds back to tell user about the current location [15] [17].

In, addition, Blind Assistant Navigation System that can help visually impaired people navigates independently at indoor environment also developed [18]. The system provides the localization by using wireless mesh network. The server will do the path planning which then communicates using wireless with the portable mobile unit. The visually impaired people can give commands and receives response from the server via audio signals using a headset with a microphone[19]. The proposed RFID technology in order to design the navigation system by providing information about their surroundings also developed. The system uses the RFID reader that mounted on end of the stick to read the transponder tags that are installed on the tactile paving[20], [21].

Besides, INSIGHT is the indoor navigation system to assist the visually impaired people to travel inside the buildings. The system used the RFID with Bluetooth technology to locate the user inside the buildings. The peopleal digital assistant (PDA) such as a mobile device used to interact with INSIGHT server and provide navigation information through voice commands. The zone that the user walked will be monitored by the system. The system will notify the user if the user travels the wrong direction [22]. The RFID network can help to determine the shortest distance from current location to the destination.
Besides the system can help to find the way back if they lost their direction and recalculate the new path [23].

In this paper, the development of navigation system which is applied to guide the visually impaired people at indoor environment. In order to provide an efficient and user-friendly navigation tools, a navigation device is developed by using passive RFID transponders which are mounted on the floor such as on tactile paving to build such RFID networks. We also equip the navigation system with digital compass to travel properly at true direction especially when turning process. The idea of positioning and localization with a digital compass and direction guiding through voice commands is implemented in this system. Some experiments are conducted which is focused on the calibration of the digital compass and relocates the visually impaired people back to the normal route if they are out of the direction. Besides, a comparison between two subjects which are human and a mobile robot is done to check the validity of the developed navigation system. As the results, the traveling speed of human and mobile robot is obtained from the experiment. This project is beneficial to visually impaired people because the navigation device designed with voice commands will help them to have better experience, safer and comfortable travel.

\section{DeVeloped NaVigation System for Visually IMPAIRED PEOPLE}

\section{A. System construction}

In order to developed the navigation system which is benefit to the visually impaired people, the total system such as a path planning system, RFID detection system, obstacle detection system is needed. However, in this paper, the developed navigation system is only one part of total navigation system which not including path planning system, obstacle avoidance system, and localization system and etc. Here, the developed system is focused on the RFID detection system and the digital compass which is used to guide the right way for visually impaired people when travel alone. Figure 1 illustrates the system configuration of one part of navigation system including the RFID detection system and the digital compass. In the developed navigation system, there are some components, is used such as RFID reader/writer module, micro-controller, voice module, Braille keypad, digital compass and etc.

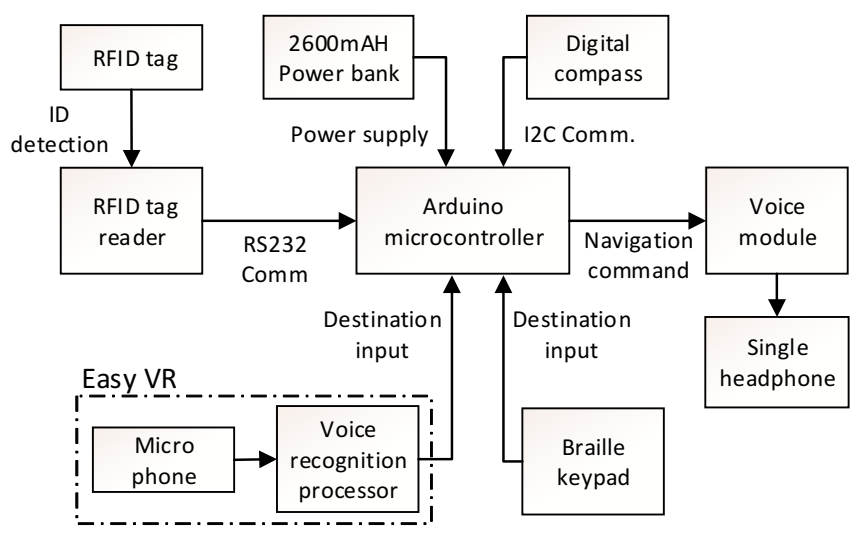

Fig. 1. System overview of developed navigation device for visually impaired people 
As the main part in the developed navigation system, a micro-controller is installed which consists memory and program in order to communicate with other peripheral. The micro-controller type which is selected to be implemented in this project is an Arduino Promini. The reason is because the Arduino Promini has been developed in small size package, light on weight and has adequate I/0 port in order to construct the navigation device. In addition, the digital compass (HMC6352) is used because it can provide the high degree heading resolution and accurate in determining the direction. If the user travels out from the path, the navigation system will determine the direction heading and gives alert to user. Once the direction is correct, the user can continue their travel by the aid of audio navigation. On the other hand, the RFID reader/writer module manufactured by Parallax also plays an important part in the developed navigation system. The RFID reader/writer module is installed at the bottom of a retractable cane for the easy detection of passive RFID tags installed on tactile paving. The RFID reader/writer module could detect the RFID transponder tags at $125 \mathrm{kHz}$ up to 3 inches distance.

Figure 2 shows the RFID detection system consists of the RFID reader/writer module. The RFID reader/writer module is directly connected to Arduino micro-controller which can be activated when the Arduino micro-controller is powered by the power supply/battery. It is also installed at the bottom of the electronic cane in order to detect the RFID tag easily which are installed on the tactile paving that can read the code of the tags and the code encryption will be done by the program inside the Arduino micro-controller. The information of the places which the RFID tags have been mounted will be prepared as a library inside the micro-controller. Each RFID tag contains pre-stored information such as the location and the surrounding environment including obstacles, place names, and building names in the library of the micro-controller with the micro SD card which has been installed with the voice module. The voice module (WTV020) is used in order to play the voice commands and inform the users which the direction that they should be taken and turn when the corner. It also played the voice command that followed by the measured value from digital compass.

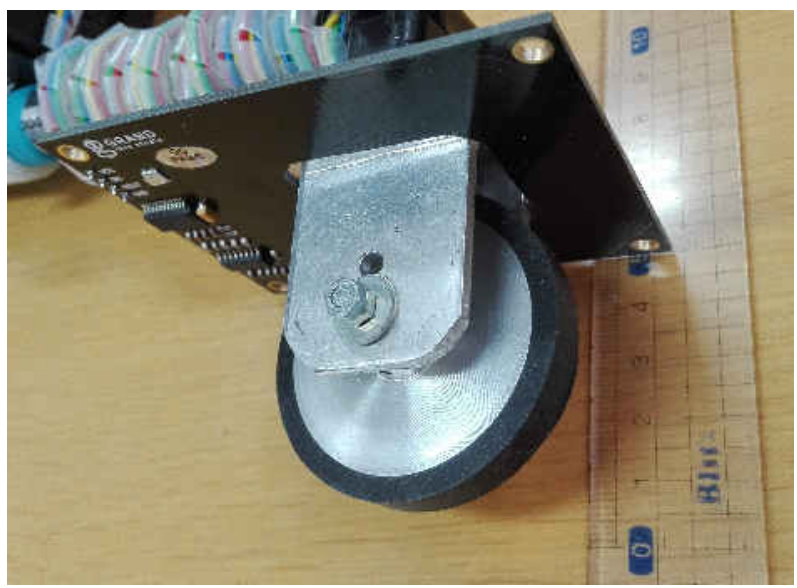

Fig. 2. Construction of RFID reader/writer module on developed electronic cane
Figure 3 shows the illustration of the electronic cane which is developed in this research. A conventional white cane is transformed to the electronic cane in order to attached all the developed system. At the bottom of the cane, a wheel is mounted in order for the RFID reader/writer module can easily detect RFID tags. The wheel size is about $4 \mathrm{~cm}$ and the RFID reader/writer module is mounted at $6 \mathrm{~cm}$ from the floor. Thus, the RFID tags can be detected respectively. At the same time, a wheel make the user does not need to raise and swing the cane while traveling which can makes the user tired to swing. If the user swing the retractable cane, the RFID tag could not be detected by the RFID reader/writer module. In addition, the retractable stick is used as the replacement of conventional white cane which is commonly used by visually impaired people for navigation purpose. The retractable stick can be shorten to $25 \mathrm{~cm}$ in order to carry and mobilize when the developed navigation device unused. Besides, the retractable stick can be extended up to maximum $120 \mathrm{~cm}$ which similar to conventional white cane. Hence, the retractable stick can be adjusted on the basis of the height and users comfortability.

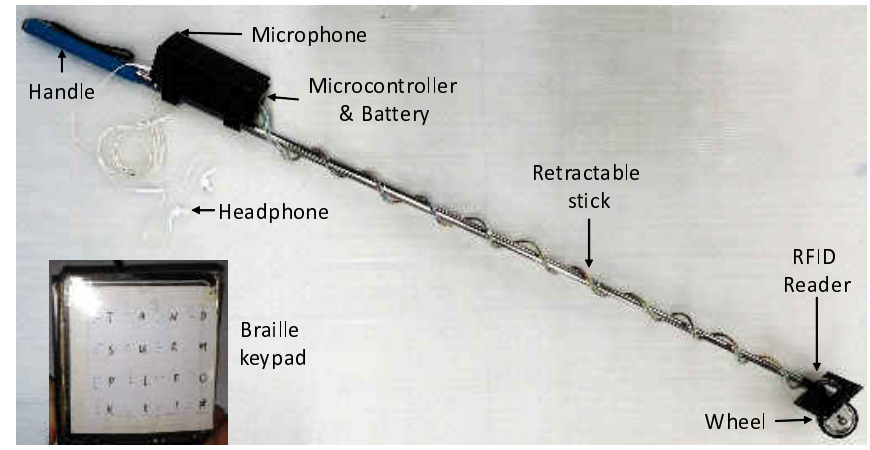

Fig. 3. Developed navigation device for visually impaired people

\section{B. Control system flow chart for navigation system}

Figure 4 shows the process flowchart of the designed and developed navigation device. In order to used the developed navigation device, the RFID reader/writer module needs to be initialized respectively. The user could set the desired destination at start point after detecting the nearest RFID tag which have been mounted on tactile paving or floor around them. In order to navigate to the desired location. The RFID reader/writer module is activated and will read the transponder tags. If the RFID reader/writer module is failed to read the tag even though the RFID tag is already in the detection range, the device will return to the tag reader activation process by initializing the RFID reader/writer module first. On the other hand, if the next RFID tags are successfully detected, the Arduino micro-controller will carry out the encryption of the tag identity. Each RFID tag has its own identification code and the code is transferred to Arduino micro-controller through RS232 for identification code encryption. After that, the microcontroller system proceeds to the navigation processing. The information extracted will be then reprocessing and converted into voice commands. Then, the users will receive the commands on how to travel their path and the information about surrounding environment. 


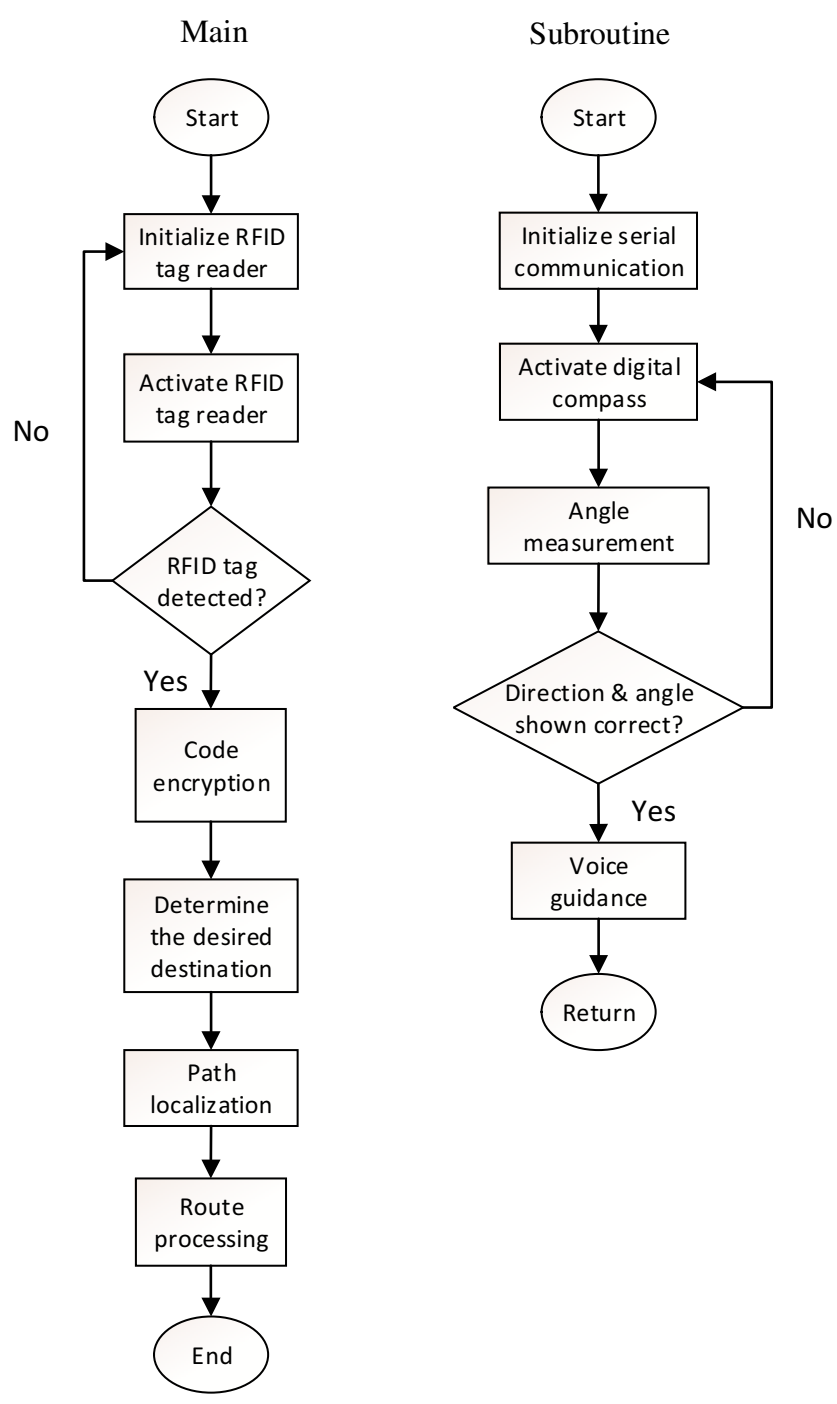

Fig. 4. Process flowchart of RFID navigation system

Once the RFID tag is detected at the starting point, the user can determine where he or she wants to go by inputting the desired location through the developed Braille keyboard or voice recognition device [24]. Figure 5 shows the modified keypad with Braille code. This Braille writing system is attached and connected on the top of the $4 \times 4$ numeric keypad. It is special modified for key in the destination with the combination alphabet " $\mathrm{T}, \mathrm{A}, \mathrm{N}, \mathrm{D}, \mathrm{S}, \mathrm{U}, \mathrm{R}, \mathrm{M}$, P, L, F, O, I, E, L, \# " which can be inputted as desired destination such as TOILET, ATM, ROOM, RESTAURANT, STORE, PLATFORM and etc. The reason why these alphabets was selected in the prototype is because a simple map just need to be applied. All the desired destination have been preprogrammed in the library of the micro-controller. The users need to key in the destination on the modified keypad with the Braille code and the device will start to give the guidance to the visually impaired people through a headphone.

After the identity encryption process is succeed, the device process to path localization will lead to user's desired destination guided by voice commands which will be given through headphone. In case the user travels at the wrong path

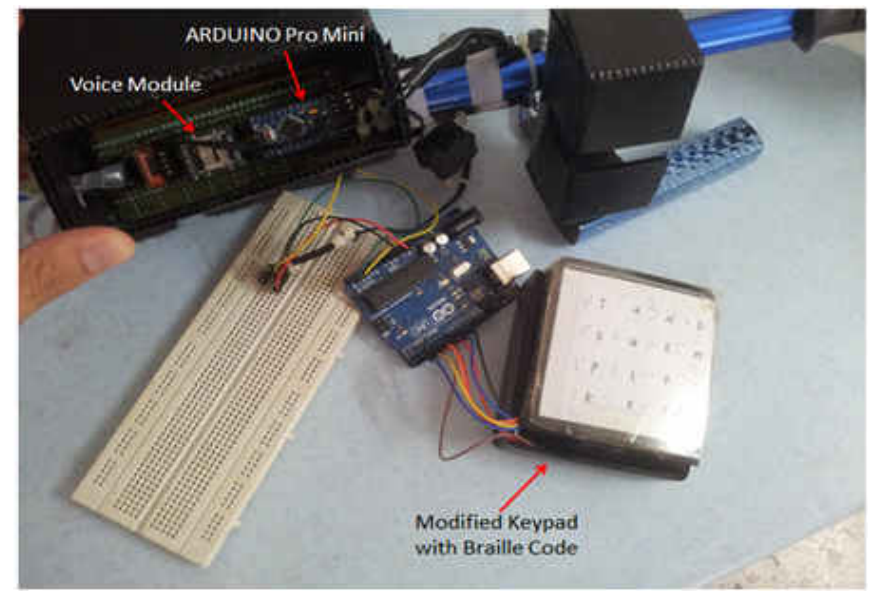

Fig. 5. The modified keypad using Braille characters

especially when the user turn at the junction, the process will proceed to route processing subroutine. In the route processing subroutine, the digital compass is activated by initializing the serial communication from the Arduino main board. The magneto sensor inside the digital compass will measure the angle deviation and recalculate the correct heading direction. If there is no more angle deviation, the user can continue his or her travel through voice commands until arrive to the destination and vice-versa.

\section{EXPERIMENTAL SETUP}

Figure 6 illustrates the ZigBee wireless networks where the communication between the server/laptop and the developed navigation device. The ZigBee network is applied to connect and monitor the developed navigation device when the experiment is conducted. The ZigBee network acts as the center of data transferring between the navigation device and the server/ laptop. The movement of user will be shown to the map processing system on the server/laptop respectively such as in Fig. 6. Hence, the user current position to the desired position will be displayed on the map based on a generated route. The map system then identifies the address of the target. Concurrently, the RFID reader/writer module will read the RFID tags on the tactile paving or floor. The data of the RFID tags of the current position and the address is sent for the map processing.

Next, voice guidance commands also will be given based on the route which have been generated to the user through an earphone. The earphone connection is based on Bluetooth connection. The server/laptop will send the voice guidance, and user position also will be updated at the same time. Path recalculation also will be done again and produces the voice guidance if the user takes the wrong path from the recommended path. The benefits of the system is when user need to take the corner turning, the digital compass will compare the angle and ensure the user to take the corner effectively without hitting the nearby obstacles. The server receives data from ZigBee network and suggests to mount at fixed locations inside the buildings. The server must be updated and the information of the destinations and objects need to be 
stored inside the database with respect to the map system.

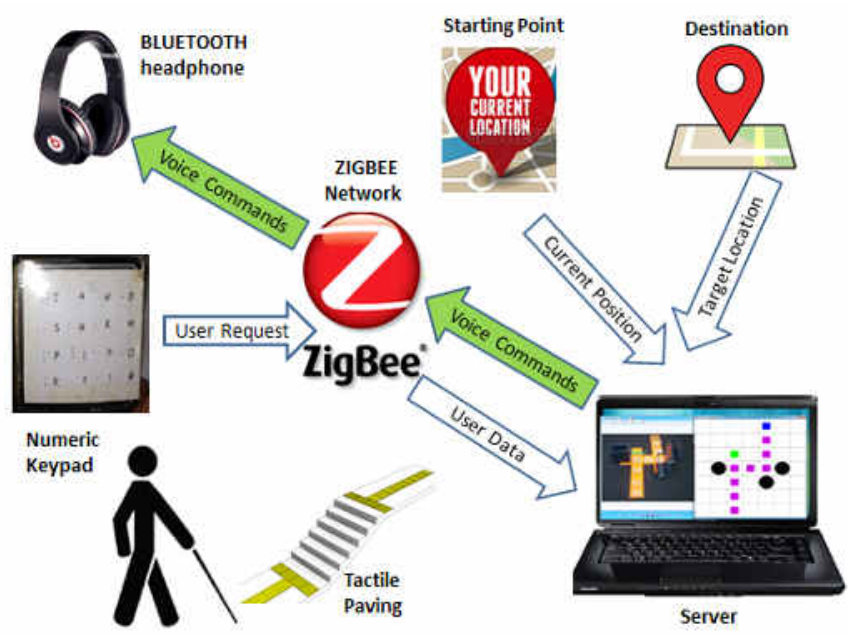

Fig. 6. System configuration including developed navigation system with server/laptop

In order to optimize the functionality of the developed navigation device for guiding the visually impaired people in the correct direction throughout the travel path, the experiment setup to evaluate the accuracy of the digital compass is set. The orientation or direction is attained by using digital compass which mounted on the developed electronic cane. Figure 7 shows the digital compass setup and the reference compass, respectively. The digital compass is connected to the Arduino Promini micro-controller to obtain the analog signal and convert it back to the digital signal by using the onboard analog digital converter (ADC). The digital signal will be displayed on the serial monitor of the Arduino Promini and the digital compass can be tuned accurately. The digital compass is fixed at the certain point where the RFID tag has been mounted to ensure the digital compass is always pointing at the north $(\mathrm{N})$. The compass which inside the iPhone is used as the reference compass in order to make comparison when calibrating the digital compass.

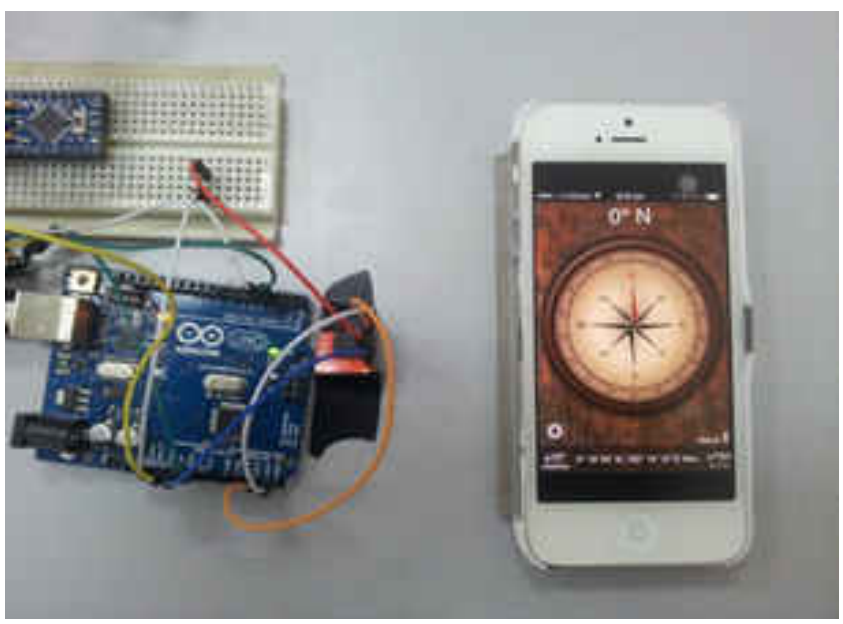

Fig. 7. Digital compass setup and the reference compass
Figure 8 shows the experimental field including tactile paving which is used for the experiment. Tactile pavings are numbered with $00,01,02,03,04,05,06$ and 07 are the points paths while blue objects represent the obstacles which is unable to pass through. Voice module (WTV020) has been saved with five types of sound which are forward, turn left, turn right, stop and warning. At this stage, only five directions of sound are used as navigation after the shortest path is generated to follow the direction from start to target node. There are two destinations which can be set in this experiment which are ATM and toilet. There are some influence factors need to be considered during the blind navigation evaluation test such as systematic error and human error.

Systematic error where there is bias in measurement lead to the path completion time. The error occurred for the time response of the system where there is some time delay for the ZigBee during data transmission and sending voice commands. Human error is another error that is not intended and cannot avoided. For this case, the human error is response of the participants when they start to walk when they received the voice commands. There are some delay at the starting point and cornering. This will give the different results for the path completion time. However, the time that is needed to complete the path does not emphasize too much on how fast the people reaches the destination.

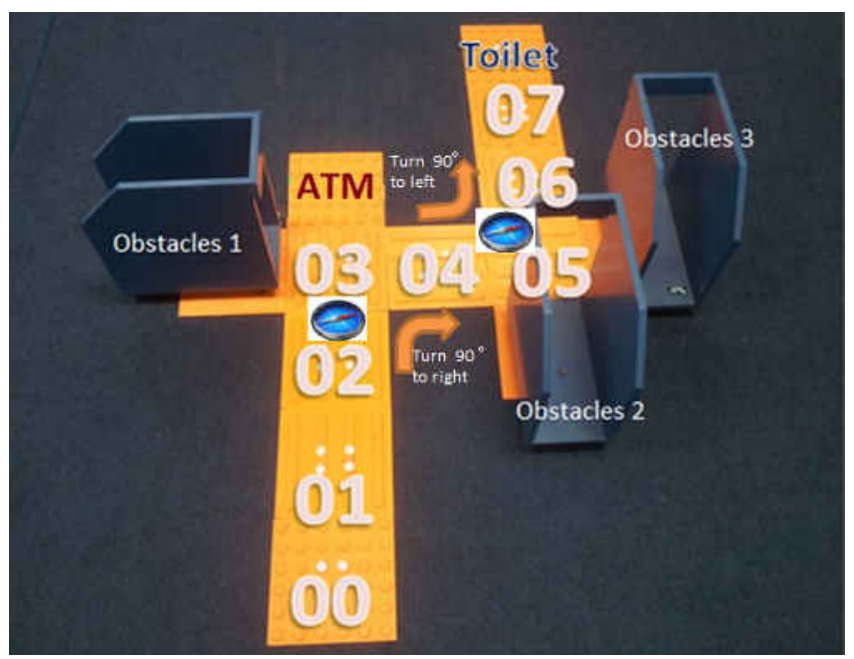

Fig. 8. Field setup which include RFID tags on tactile paving with some obstacles

\section{EXPERIMENTAL RESULTS}

\section{A. Performances of compass technology in navigation system}

Table I shows the digital compass accuracy test results when the digital compass is pointing to north $(\mathrm{N})$. From the table, the digital compass measurement clearly shows that the resolution of the digital compass is high and able to produce the digital compass reading in two significant values. The repeatability test is carried out about 20 times to proof that the results are valid to be used in the navigation device. Besides, the accuracy test for the digital compass have been done 20 times at different places and different time. The relative error is getting smaller and close to the north $(\mathrm{N})$ direction which 
is shown in the result because the digital compass output are getting stable. This implies that the digital compass is suitable to use for blind navigation to provide accurate heading direction.

TABLE I. COMPass ACCURACy TEST RESUlt

\begin{tabular}{ccccc}
\hline Times & $\begin{array}{c}\text { Pointing to } \\
\text { North }(\mathrm{N})\end{array}$ & $\begin{array}{c}\text { Degrees } \\
\text { North(N) }\end{array}$ & $\begin{array}{c}\text { Relative } \\
\text { error }\end{array}$ & $\begin{array}{c}\text { Percentage } \\
\text { relative error }\end{array}$ \\
\hline 1 & Yes & 356.30 & 3.70 & 1.0278 \\
2 & Yes & 356.40 & 3.60 & 1.0000 \\
3 & Yes & 356.30 & 3.70 & 1.0278 \\
4 & Yes & 355.70 & 4.30 & 1.1944 \\
5 & Yes & 358.20 & 1.80 & 0.5000 \\
6 & Yes & 357.80 & 2.20 & 0.6111 \\
7 & Yes & 357.70 & 2.30 & 0.6389 \\
8 & Yes & 357.80 & 2.20 & 0.6111 \\
9 & Yes & 358.20 & 1.80 & 0.5000 \\
10 & Yes & 357.90 & 2.10 & 0.5833 \\
11 & Yes & 359.20 & 0.80 & 0.2222 \\
12 & Yes & 359.30 & 0.70 & 0.1944 \\
13 & Yes & 359.20 & 0.80 & 0.2222 \\
14 & Yes & 359.30 & 0.70 & 0.1944 \\
15 & Yes & 359.30 & 0.70 & 0.1944 \\
16 & Yes & 359.20 & 0.80 & 0.2222 \\
17 & Yes & 359.20 & 0.80 & 0.2222 \\
18 & Yes & 359.60 & 0.40 & 0.1111 \\
19 & Yes & 359.40 & 0.60 & 0.1666 \\
20 & Yes & 359.40 & 0.60 & 0.1666 \\
\hline
\end{tabular}

Note : North(N) direction point to $0^{\circ}$ or 360

Mean of 20 times repeatability $=358.27^{\circ}$

Mean of percent relative error $=0.4805 \%$

Figure 9 shows the percent relative error $\%$ of the readings obtained from the digital compass when it is pointing to the north $(\mathrm{N})$. The maximum peak of the percent relative error is $1.1944 \%$. The average percent relative error is $0.4805 \%$. The graph shows the percent relative error is decreasing gradually towards the end and becomes nearly constant between the 11 and 17 times of trials. This implies that the relative error is getting smaller and the readings are very close to $360^{\circ}$ when the digital compass points to the north $(\mathrm{N})$. The obtained data is said to have high reliability. Besides, the digital compass has very high sensitivity and able produces significant value at tenth decimal places.

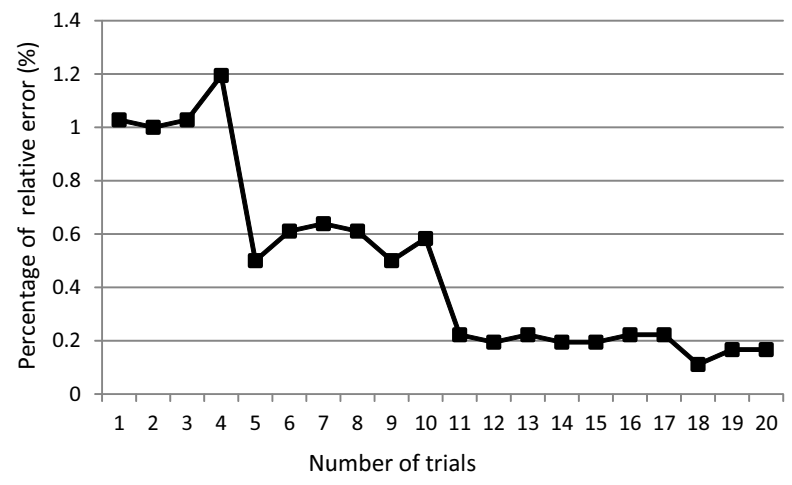

Fig. 9. Percentage of relative error for digital compass calibration when pointing to north

\section{B. Performance of developed navigation system in field test}

From the table which shown the validity of the digital compass which can be used inside the developed navigation system, the developed navigation system is evaluated for its performance at the real field by using the RFID tag that have been installed on tactile paving. The navigation device built with the digital compass for direction guidance and voice module able to inform user about the direction. The digital compass will be able to detect the error if the user travels out of the direction and the misdirection lead to the wrong path. Thus, the voice module will inform the user, "WARNING!" repeatedly and alert user from taking the wrong path. The navigation proceeds until the user turn over and travel on the right direction. Figure 10 shows the illustration of the developed navigation device which is conducted on different subjects such as human and mobile robot.

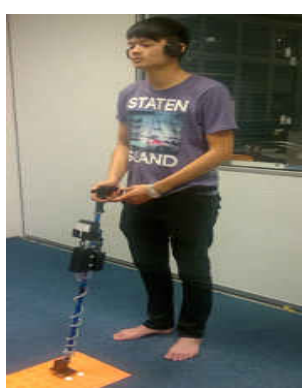

(a) Human

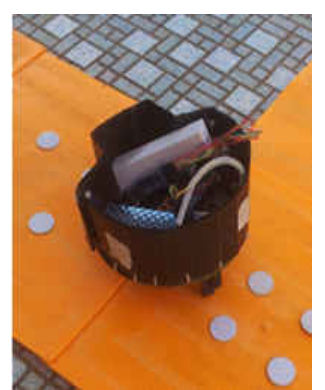

(b) Mobile robot
Fig. 10. Experiment conducted for comparing the performance of navigation device based on different subjects such as human and mobile robot

Here, the traveling time which is consumed to finished the route is measured. The travel distance which is needed to be done is $240 \mathrm{~cm}$ which each tactile paving about $30 \mathrm{~cm}$ in length. There are two subjects which have been tested at the field which are human and mobile robot. The RFID reader/writer module is attached at the bottom of the mobile robot in order to easily detect the RFID tag. Then, RFID reader/writer module reads the tags first and at the same time the Arduino Promini micro-controller will synchronize with the digital compass for route processing. For experiment by using a mobile robot, the command are given to the DC motor in order to go straight or turn. However, for human, the voice module will inform the user how to turn at the corner, e.g. "90 degree turn left" or "90 degree turn right". Through this experiment, the high accuracy of the digital compass is able to give direction fast and precise.

Table II shows the average traveling time which is recorded for mobile robot is $25 \mathrm{~s}$ while for human is $33 \mathrm{~s}$. From these results, the difference which can be related is the size of the subject. The mobile robot which is used are wheeled robot which sized $20 \mathrm{~cm}$ in diameter. However, the human subject which are tested in this experiment are $170 \mathrm{~cm}$ in height and the position of human is $40 \mathrm{~cm}$ behind the end of the electronic cane. Therefore, the human subject is quite difficult to turn when the voice command is given. Meanwhile, the mobile robot is easily turn because the mobile robot can turn at the same axis when the command which are given to the DC motor, respectively.

Figure 11 shows the picture from the video for human when 
the experiment is conducted. These pictures is taken at every second for all time elapsed which take 54 s to complete the course that have been set. In order to set the desired destination which is TOILET by using the developed Braille keypad, the human subject took about 21 s from the starting point. Then, the human subject took about $33 \mathrm{~s}$ to travel from the starting point to the desired destination. The human subject travelled by using voice guidance from the developed navigation device such as forward, turn right, turn left, and etc.

TABLE II. PERFORMANCE COMPARISON OF DIFFERENT SUBJECTS

\begin{tabular}{ccc}
\hline Subject & Human with electronic cane & Mobile robot \\
\hline Size & $170 \mathrm{~cm}$ (Height) & $20 \mathrm{~cm}$ (Diameter) \\
Average travelled time & $33 \mathrm{~s}$ & $25 \mathrm{~s}$ \\
\hline
\end{tabular}

\section{CONCLUSION}

In this paper, the navigation device for visually impaired people has been developed and some experiments has been evaluated. The digital compass which is applied can provide accurate direction which help to guide the visually impaired while travel independently. The RFID detection system is beneficial to the visually impaired people since these people provided by feedback information about the current location and the surrounding obstacles. Besides, the performance evaluation for the human and robot subject also successfully done.

Since the developed navigation device was the earlier stage of development, the implementation of the shortest path algorithm will be applied to search the shortest route in the future. The blind navigation device with RFID technology supported by shortest path algorithm will be conducted to ensure them to have a better and comfortable travel at the indoor environment. Besides, the design of the navigation device also will be improved in terms of weight and sustainability design concept.

\section{ACKNOWLEDGMENT}

This research is a collaboration project between Tokushima University and Universiti Teknikal Malaysia Melaka. This research also was supported by research grant from Universiti Teknikal Malaysia Melaka award no. FRGS/1/2014/TK03/FKE/03/F00213.

\section{REFERENCES}

[1] World Health Organization, "WHO - Visual Impairment and Blindness." Fact Sheet No. 282, 2014

[2] J. Borenstein and I. Ulrich, "The GuideCane-a computerized travel aid for the active guidance of blind pedestrians," Proceedings of International Conference on Robotics and Automation, vol. 2, pp. 12831288, 1997.

[3] S. Shoval, I. Ulrich, and J. Borenstein, "NavBelt and the Guide-Cane [obstacle-avoidance systems for the blind and visually impaired]" IEEE Robotics and Automation Magazine, vol. 10, no. 1, pp. 9- 20, 2013

[4] A.M Kassim, Jamaluddin, M.H., Yaacob, M.R., Anwar, N.S.N., Sani, Z.M., Noordin, A., "Design and development of MY 2nd EYE for visually impaired people " 2011 IEEE Symposium on Industrial Electronics and Applications (ISIEA), pp. 700-703, 2011

[5] A.M Kassim, M.S Jamri, M.S.M Aras, M.Z.A Rashid, MR Yaacob, "Design and development of obstacle detection and warning device for above abdomen level " 2012 12th International Conference on Control, Automation and Systems (ICCAS), pp. 410-413, 2012

[6] A.M Kassim, A.Z Shukor, C.X Zhi, T Yasuno, "Performance Study of Developed SMART EYE for Visually Impaired people " Australian Journal of Basic and Applied Sciences, 7 (14) pp. 633-639, 2013
[7] V. Kulyukin, C. Gharpure, and J. Nicholson, "RoboCart: toward robotassisted navigation of grocery stores by the visually impaired," 2005 IEEE/RSJ International Conference on Intelligent Robots and Systems, pp. 2845-2850, 2005.

[8] E. Wise et al., "Indoor Navigation for the Blind and Vision Impaired: Where are we and where are we going?, "2012 International Conference on Indoor Positioning and Indoor Navigation (IPIN), pp. 1-7, 2012

[9] U. Biader Ceipidor, C.M. Medaglia, F.Rizzo, A.Serbanati, "Radio Virgilio/Sesamonet: an RFID-based Navigation system for visually impaired " pp.1-6,

[10] Jaime Sanchez, "Mobile Audio Navigation Interfaces for the Blind ", Universal Access in Human-Computer Interaction. Intelligent and Ubiquitous Interaction Environments, Vol. 5615 of the series Lecture Notes in Computer Science, pp. 402-411, 2009

[11] Lisa Ran, Sumi Helal and Steve Moore, "Drishti: An Integrated Indoor/Outdoor Blind Navigation System and Service " Proceedings of the Second IEEE Annual Conference on Pervasive Computing and Communications, PerCom 2004, pp. 23-30, March 2004

[12] M. Sarfraz and S. A. J. Rizvi, " Indoor Navigational Aid System for the Visually Impaired," Geometric Modeling and Imaging (GMAI), pp. 127-132, 2007

[13] S. S. Santhosh, T. Sasiprabha, and R. Jeberson, "BLI-NAV embedded navigation system for blind people, "Recent Advances in Space Technology Services and Climate Change (RSTSCC) 2010, pp. 277-282, 2012

[14] M. H. Choudhury, D. Aguerrevere, and A. B. Barreto, "A Pocket-PC Based Navigational Aid for Blind Individuals," 2004 IEEE Symposium on Virtual Environments, Human-Computer Interfaces and Measurement Systems (VECIMS), pp. 43-48, 2004

[15] M. Bousbia-Salah, A. Redjati, M. Fezari, and M. Bettayeb, "An ultrasonic navigation system for blind people," 2007 IEEE International Conference on Signal Processing and Communications ICSPC, pp. 1003-1006, 2007

[16] A.M Kassim and M.S Jamri and M.S.M Aras and M.Z.A Rashid, "Design and Development of Vibration Method for Vehicle Reverse System (VRS) " Journal of Procedia Engineering, vol. 41, pp. 11141120, 2012

[17] M.R Yaacob, N.S.N Anwar, A.M Kassim , “Effect of Glittering and Reflective Objects of Different Colors to the Output Voltage-Distance Characteristics of Sharp GP2D120 IR" ACEEE International Journal on Electrical and Power Engineering. 3 (2). pp. 6-10, 2012

[18] M. Shamsi, M. Al-Qutayri, and J. Jeedella, "Blind assistant navigation system, " 2011 1st Middle East Conference on Biomedical Engineering (MECBME), pp. 163-166, 2011

[19] S. Chumkamon, P. Tuvaphanthaphiphat, and P.Keeratiwintakorn, (2008) "A blind navigation system using RFID for indoor environments," 5th International Conference on Electrical Engineering/ Electronics, Computer, Telecommunications and Information Technology, vol. 2, pp. 765-768.

[20] A.M. Kassim, H. I Jaafar, M.A. Azam, N. Abas, T.Yasuno, "Performances study of distance measurement sensor with different object materials and properties" 3rd IEEE International Conference on System Engineering and Technology (ICSET), pp. 281-284, 2013

[21] A.M. Kassim, H. I Jaafar, M.A. Azam, N. Abas, T.Yasuno, "Design and Development of Navigation System by using RFID Technology" 3rd IEEE International Conference on System Engineering and Technology (ICSET), pp. 258-262, 2013

[22] A., Gandhi, S. R., Wilson, C., and Mullett, G., "INSIGHT: RFID and Bluetooth enabled automated space for the blind and visually impaired" IEEE International Conference of Engineering in Medicine and Biology Society, pp. 331-334, 2010

[23] A.M Kassim, A.Z Shukor, C.X Zhi, T Yasuno, "Exploratory Study on Navigation System for Visually Impaired people” Australian Journal of Basic and Applied Sciences, 7 (14) pp. 211-217, 2013

[24] Anuar bin Mohamed Kassim, Takashi Yasuno, Hazriq Izzuan Jaafar, Mohd Aras Mohd Shahrieel, "Development and Evaluation of Voice Recognition Input Technology in Navigation System for Blind people”, Journal of Signal Processing, Vol.19, No.4, pp.135-138, July 2015 

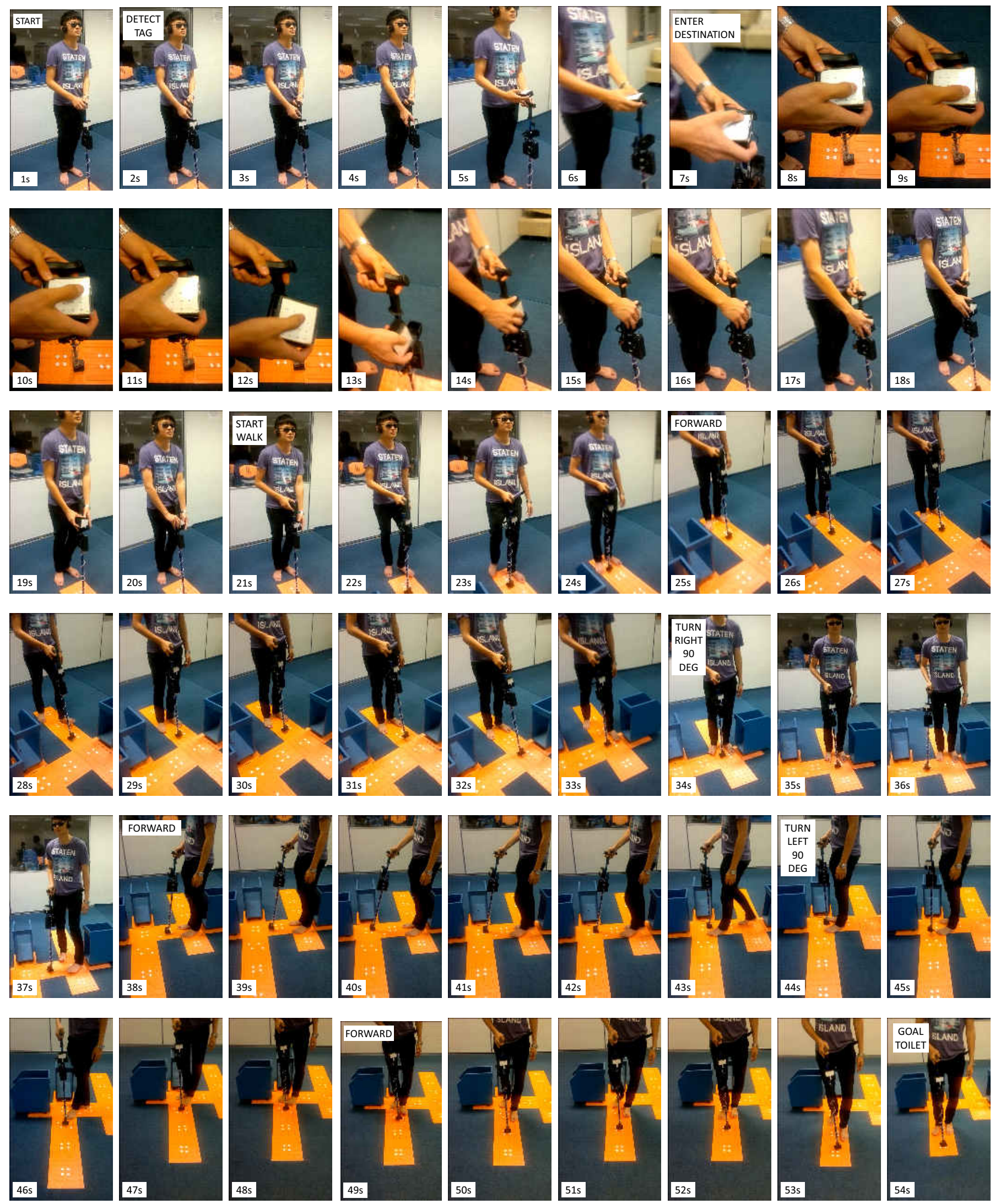

Fig. 11. Pictures for each second by human subject travelled using developed navigation device with voice guidance 